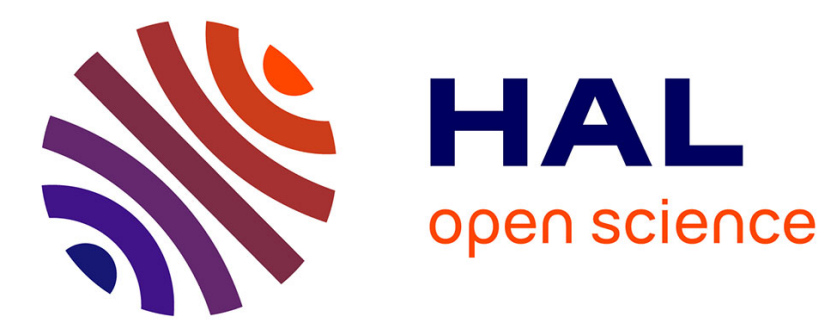

\title{
Diagnostic des systèmes dynamiques linéaires sans modèle explicite
}

\author{
Abdouramane Moussa Ali, Cédric Join, Frédéric Hamelin
}

\section{To cite this version:}

Abdouramane Moussa Ali, Cédric Join, Frédéric Hamelin. Diagnostic des systèmes dynamiques linéaires sans modèle explicite. Journal Européen des Systèmes Automatisés (JESA), 2011, 45 (Spécial: Nouvelles tendances en automatique), pp.339-362. hal-00580303v2

\section{HAL Id: hal-00580303 \\ https://hal.science/hal-00580303v2}

Submitted on 9 Dec 2011

HAL is a multi-disciplinary open access archive for the deposit and dissemination of scientific research documents, whether they are published or not. The documents may come from teaching and research institutions in France or abroad, or from public or private research centers.
L'archive ouverte pluridisciplinaire HAL, est destinée au dépôt et à la diffusion de documents scientifiques de niveau recherche, publiés ou non, émanant des établissements d'enseignement et de recherche français ou étrangers, des laboratoires publics ou privés. 


\title{
Diagnostic des systèmes dynamiques linéaires sans modèle explicite
}

\author{
A. MOUSSA ALI* - C. JOIN*** - F. HAMELIN* \\ * CRAN, Nancy-Université, UMR 7039 CNRS, BP 70 239, 54506 Vandouvre, France \\ ** ALIEN-INRIA Saclay - Ile de France
}

\begin{abstract}
RÉSUMÉ. Nous proposons une méthode de diagnostic des défauts actionneur et capteur modélisés au travers de signaux structurés sur une classe particulière de systèmes dynamiques linéaires incertains. Le principal atout de cette approche est qu'il est possible, sous certaines hypothèses, de détecter, localiser et identifier les défauts à l'aide des seules mesures de la commande et de la sortie sans avoir à identifier les paramètres du modèle. La méthode est fondée sur la génération et l'analyse de relations de redondance analytique et exploite le fait qu'un signal structuré satisfait une équation différentielle. La prise de décision est entièrement fondée sur l'évolution temporelle des estimations de certaines caractéristiques des défauts. Un exemple numérique est fourni et commenté afin d'illustrer l'approche proposée.

ABSTRACT. We propose a diagnosis approach of sensor and actuator modeled as structured signals acting on a particular class of uncertain linear dynamical systems. The main advantage of this approach is that it is possible under certain assumptions, to detect, isolate and identify faults using only input and output measurements without having to identify model parameters. The method is based on the generation and analysis of analytical redundancy relations and exploits the fact that a structured signal satisfies a differential equation. The decision rule is based entirely on the temporal behaviour of the estimates of some fault characteristics. A numerical example is provided and discussed to illustrate the proposed approach.

MOTS-CLÉS : Théorie des distributions, analyse spectrale, défaut actionneur, défaut capteur, FDI. KEYWORDS: distribution theory, spectra analysis, actuator fault, sensor fault, FDI.
\end{abstract}

JESA - 45/2011. Nouvelles tendances en automatique 


\section{Notations utilisées}

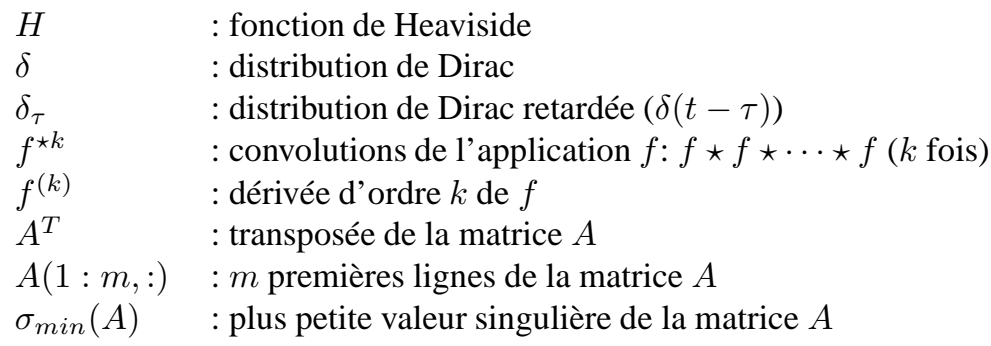

\section{Introduction}

Comme dans son acceptation médicale, le diagnostic des systèmes automatiques consiste à remonter des symptômes perçus vers les causes. Il est également défini comme l'ensemble des actions mises en œuvre en vue de détecter, localiser et identifier tout phénomène anormal sur un système à partir de la connaissance disponible sur ce dernier. La connaissance disponible inclut, non seulement, les sorties du contrôleur (qui a pour but d'améliorer les performances du système), les mesures fournies par les capteurs mais aussi, le plus souvent, des informations sur la structure et/ou un modèle du système. Parmi les méthodes classiques de diagnostic existant dans la littérature, il existe celles à base de modèle explicite du système qui sont théoriquement bien établies ((Frank, 1990), (Basseville et al., 1993), (Patton et al., 2000), (Isermann, 2006), (Ding, 2008)). Cependant, rappelons que la fiabilité donnée par le modèle explicite est en général accompagnée par l'inconvénient d'une trop grande complexité. Ce modèle peut ne pas être utilisable en pratique et il est souvent nécessaire d'en réduire la complexité.

C'est ainsi qu'est introduite la notion d'approches intégrées considérant simultanément l'identification et le diagnostic. L'une des approches intégrées les plus étudiées et ayant du succès dans les applications industrielles est l'approche de diagnostic par analyse en composantes principales notée ACP ((Gertler et al., 2004), (Ding et al., 2010)). Une limitation importante de ces approches est que le modèle ACP, une fois élaboré à partir des données, est stationnaire (invariant dans le temps), alors que la plupart des systèmes réels présentent généralement des comportements variant légèrement avec le temps (dus au vieillissement par exemple). En plus l'identification, de manière implicite, des matrices du système fournie par l'approche ACP (Wang et al., 2002) comporte des incertitudes paramétriques rendant indispensable la condition de persistance d'excitation pour la génération de résidus robustes à ces incertitudes.

Pour faire face aux difficultés rencontrées dans les approches de diagnostic par $\mathrm{ACP}$ et celles à base de modèles explicites en général, une nouvelle méthode algébrique de diagnostic de système en présence de défaut actionneur et/ou capteur est proposée dans cet article. Cette approche présente un nouveau point de vue basé sur la théorie des distributions et l'analyse du pseudospectre de faisceaux de matrices. Notre 
démarche s'inspire de certains outils et développements de la théorie de l'estimation algébrique (Fliess et al., 2003) courants en automatique mais très peu usuels en traitement du signal. Cette approche repose uniquement sur la connaissance structurelle du système ainsi que des signaux défauts. Plus précisément on considère les systèmes régis par une équation différentielle linéaire à coefficients constants et des défauts actionneur et capteur additifs dits structurés.

On adopte pour le développement de notre approche des formulations distributionnelles (également utilisées dans (Belkoura, 2010)) grâce auxquelles, d'une part, nous obtenons des expressions explicites dans le domaine temporel et d'autre part, nous pouvons transformer le problème de détection de défauts en un problème de recherche de valeurs propres généralisées, à partir duquel ni la connaissance, ni l'estimation des paramètres du modèle ne sont nécessaires pour la détection de défauts.

L'une des propriétés importantes et hautement souhaitables pour un système de diagnostic est sa réactivité et sa précision. Or dans une approche stochastique, la connaissance a priori d'informations statistiques du bruit et/ou des signaux traités est très importante. En plus la performance de nature asymptotique avec les retards de traitement sont inhérents à une approche stochastique. Dans ce contexte une approche algébrique et déterministe dévient intéressante. Même si le cadre déterministe est souvent déconsidéré essentiellement en raison de la sensibilité des méthodes aux bruits, une telle approche peut se révéler efficace lorsqu'elle est abordée dans un cadre théorique approprié.

La méthode proposée exploite les caractéristiques des signaux en continu. Comme nous le verrons, la connaissance des signaux entrée et sortie traités en temps continu permet le diagnostic de défaut sur une fenêtre de temps très courte. De plus, la rapidité des estimations (méthode non asymptotique), liée aux formules explicites fournies par les techniques algébriques peut être un atout majeur pour l'accommodation des défauts (Staroswiecki et al., 2000).

En revanche, non seulement les méthodes déterministes ont besoin de la connaissance de l'ordre du système, mais en plus elles s'y montrent très sensibles.

L'article est organisé comme suit. La section 2 est consacrée aux rappels des outils algébriques nécessaires au développement de la méthode. Dans la section 3, on illustre les principales étapes de la méthode à travers des exemples simples avec des systèmes d'ordre un. Dans la section 4, on met en évidence certaines robustesses de l'approche, par rapport à l'ordre du système et aux bruits de mesure.

\section{Base algébrique de l'approche}

\subsection{Rappel sur les distributions}

Le formalisme des distributions offre une grande souplesse et un cadre unifié dans l'énoncé et le traitement des différents problèmes de modélisation des systèmes linéaires et stationnaires. Dans cet article une formulation distributionnelle est adoptée 
et quelques notions fondamentales pour l'approche de diagnostic proposée sont rappelées.

Une distribution est une fonctionnelle linéaire, continue et définie sur l'ensemble des fonctions régulières à support (partie du domaine de définition sur laquelle se concentre l'information utile) compact (fermé et borné). On dit qu'une distribution s'annule sur un ensemble $\Omega$ si elle s'annule sur toutes les fonctions à support inclus dans $\Omega$. Le complémentaire du plus grand ouvert sur lequel $T$ est identiquement nulle s'appelle support de $T$ (noté $\operatorname{Supp}(T)$ ). On définit aussi l'ordre d'une distribution $T$ (noté $\operatorname{Ordre}(T)$ ) comme le plus petit entier naturel $m$, s'il existe, tel que : $\exists C>0$, pour toute fonction régulière $\phi$, on a $|T(\phi)| \leq C$ sup $\quad\left\|\phi^{(i)}\right\|_{\infty}$

Exemple $1 \operatorname{Ordre}\left(\delta_{\tau}\right)=0$ et $\operatorname{Supp}\left(\delta_{\tau}\right)=\{\tau\}$.

La théorie des distributions étend la notion de dérivée à toutes les fonctions localement intégrables. Si la fonction $f$ est une fonction continue et dérivable, excepté au point $x$ où elle présente un saut fini $s_{x}$, alors la dérivée de la distribution associée à $f$ est donnée par $\dot{f}-s_{x} \delta_{x}$, avec $\dot{f}$ la dérivée usuelle de $f$ (définie sur $\mathbb{R} /\{x\}$ ). Les opérations de dérivation, d'intégration et de translation peuvent être traduites par des produits de convolution :

$$
\dot{y}=\delta^{(1)} * y, \int_{0}^{t} y(\tau) d \tau=H * y, y(t-\tau)=\delta_{\tau} * y
$$

Le théorème qui suit est le résultat principal sur lequel se fonde la méthode de détection et localisation de défauts présentée.

Théorème 1 (Schwartz, 1966)

La multiplication d'une distribution $T$ à support $S u p p(T)$ compact et d'ordre fini $m$ par une fonction régulière $\alpha$ s'annulant ainsi que ses dérivées jusqu'à l'ordre $m$ sur Supp $(T)$, est une distribution nulle (i.e $\alpha T=0$ ).

L'application de ce théorème permet d'obtenir

$$
(t-\tau)^{k} \delta_{\tau}^{(n)}= \begin{cases}0 & \text { si } k>n \\ (-1)^{k} \frac{n !}{(n-k) !} \delta_{\tau}^{(n-k)} & \text { sinon }\end{cases}
$$

Enfin on a, pour toutes distributions $S$ et $T$ (dont la convolution est bien définie) et tout $t \in \mathbb{R}, n \in \mathbb{N}$, l'égalité suivante :

$$
t^{n}(S * T)=\sum_{k=0}^{n} C_{n}^{k}\left(t^{k} S\right) *\left(t^{n-k} T\right)
$$




\subsection{Signaux structurés et annihilateurs}

Les signaux structurés sont définis pour faire référence aux signaux pouvant être annulés au moyen de simples multiplications et dérivations. C'est le cas par exemple des signaux solutions d'une équation différentielle. Un exemple simple est un biais actionneur modélisé par le signal échelon $f(t)=l H(t-\tau)$. En effet, on vérifie que

$$
(t-\tau) f^{(1)}=0 \quad \forall t
$$

Dans ce contexte, l'opérateur différentiel

$$
D=t \frac{\partial}{\partial t}-\tau_{a} \frac{\partial}{\partial t}=\left(t-\tau_{a}\right) \frac{\partial}{\partial t}
$$

est appelé annihilateur ou annulateur de $f$ et il n'est pas unique.

Définition 1 On dit qu'un signal $f(t)$ est structuré s'il existe un polynôme différentiel $P \in \mathcal{E}^{\prime}(\mathbb{R})$ tel que le produit de convolution de ce dernier par $f$ résulte en une distribution $Q$ de support discret et fini contenu dans $\mathbb{R}^{+}\left(c-\grave{a}-d Q \in D_{+}^{\prime}(\mathbb{R})\right.$ et $\left.P \star f=Q\right)$.

Un annulateur correspondant au signal $f$ (lorsqu'il est structuré) est donné par

$$
D(t)=\alpha(t) Q
$$

avec $\alpha(t)$ une fonction qui satisfait avec la distribution $Q$ (d'ordre fini et à support compact) les conditions du théorème (11). La fonction $\alpha$ dépend seulement de la structure de la distribution $Q$. Lorsque $Q$ admet une décomposition du type $Q=\sum Q_{i}$ avec $Q_{i}$ des distributions de supports disjoints, alors la fonction $\alpha$ consiste au produit $\prod \alpha_{i}$ avec $\alpha_{i}$ vérifiant l'égalité $\alpha_{i} Q_{i}=0$.

L'annulation d'un terme structuré n'implique pas nécessairement la perte de toute information sur ce terme et l'annulateur peut être choisi afin de conserver une information particulière sur le signal considéré. Par exemple, en [3] l'annulateur comporte l'instant de saut $\tau-a$ du signal considéré.

\subsection{Modèles de défauts}

Une modélisation convenable (appropriée) des défauts est importante pour le bon fonctionnement des méthodes de détection de défauts. Une approche réaliste présuppose la connaissance du lien entre les défauts physiques et leur effet sur les modèles mathématiques du procédé. Ceci peut habituellement être fourni par l'inspection du système, la connaissance de sa physique et une analyse de l'arbre de symptôme de chaque défaut. Les défauts peuvent être de diverses sources. Ils peuvent être issus, par exemple

1) d'erreurs de conception, erreurs d'assemblage ; 
2) d'un fonctionnement erroné, manque de maintenance ;

3) d'un vieillissement, dégradation, usure pendant le fonctionnement normal.

En considérant la phase de fonctionnement, ils peuvent être déjà présents ou peuvent apparaître soudainement avec une petite ou grande amplitude, par étape, ou graduellement comme une dérive. Ils sont considérés comme défauts déterministes. Spécialement désavantageux, sont des défauts intermittents qui apparaissent comme des défauts stochastiques.

Un défaut est défini comme étant une déviation non permise d'au moins une propriété caractéristique d'une condition (nominale, usuelle). Les caractéristiques les plus couramment utilisées sont l'entrée $u(t)$ et la sortie $y(t)$ du système. Ainsi, l'apparition d'un défaut peut se traduire comme la modification d'une variable $x_{r}(t)$ par addition de défaut $f(t)$ :

$$
x(t)=x_{r}(t)+f(t)
$$

L'évolution temporelle de tel défaut peut apparaître comme un

1) défaut brusque (biais d'actionneur);

2) défaut naissant (dérive de capteur) ;

3) défaut intermittent (avec interruptions).

que l'on peut modéliser à l'aide de signaux structurés.

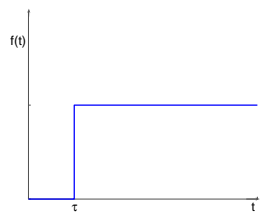

(a) défaut brusque

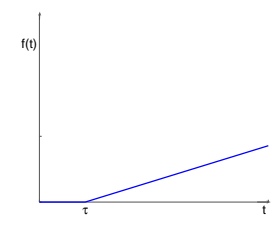

(b) défaut naissant

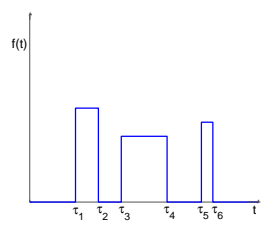

(c) défaut intermittant

Figure 1. Évolution temporelle de différents types de défauts

Exemple 2 Défauts de capteurs

Les capteurs sont des éléments dynamiques de transfert pour lesquels seule la sortie $y(t)$, la variable de mesure, est accessible. La vraie entrée physique $y_{r}(t)$ est le plus souvent inconnue.

La caractérisation du type de défaut (additif ou multiplicatif) dépend du modèle et de la nature physique utilisés du défaut. Plusieurs défauts capteurs peuvent être modélisés comme défauts additifs. Principalement, on distingue deux défauts capteur différents :

1) décalage constant, par exemple $y=y_{r}+\Delta y H\left(t-\tau_{c}\right)$;

2) décalage évolutif, par exemple $y=y_{r}+\Delta y\left(t-\tau_{c}\right) H\left(t-\tau_{c}\right)$. 


\section{Principales étapes de la méthode}

\subsection{Préliminaire}

Le système étudié est le système $\left(\sum\right)$ représenté sur la figure 2. Le but est de détecter, localiser et identifier les défauts $f_{a}$ et $f_{c}$, modélisés comme signaux structurés (Fliess et al., 2003), à l'aide de la connaissance des signaux d'entrée $u(t)$ et de sortie $y(t)$. Dans un premier temps, nous ne considérons pas l'addition du bruit. Ces types de signaux recouvrent une grande plage de signaux défauts que l'on retrouve dans la littérature (rupture, défaut naissant ou intermittent) (Isermann, 2006).

Pour diagnostiquer les défauts sur le système, on va supposer que la commande $u_{r}$ (reçue en entrée par le système) et la sortie $y_{r}$ (délivrée en sortie par le système) satisfont l'équation différentielle linéaire suivante :

$$
\left\{\begin{array}{l}
a_{n} y_{r}^{(n)}+a_{n-1} y_{r}^{(n-1)}+\cdots+a_{0} y_{r}=b_{z} u_{r}^{(z)}+b_{z-1} u_{r}^{(z-1)}+\cdots+b_{0} u_{r} \\
y_{r}^{(i)}(0), i=0, \cdots, n-1
\end{array}\right.
$$

où $n, z$ sont supposés connus et les paramètres $a_{i}, b_{j}$ ainsi que les conditions initiales sont supposés constants mais pas nécessairement connus. Sans perte de généralité, nous considérons le plus souvent le cas $z=0$.

En présence d'un défaut actionneur noté $f_{a}$ et d'un défaut capteur noté $f_{c}$, les mesures $u$ et $y$ sont explicitées en fonction des grandeurs vraies et des défauts comme suit : $u=u_{r}-f_{a}$ et $y=y_{r}+f_{c}$ (figure 2).

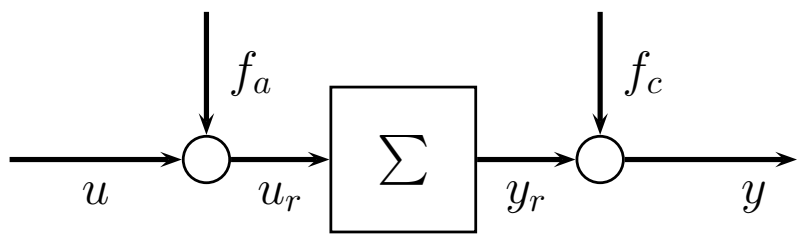

Figure 2. Système avec défaut actionneur et défaut capteur

\subsection{Système avec défaut actionneur}

Considérons un cas très simple, où $n=1$ et avec uniquement l'hypothèse d'un défaut actionneur $f_{a}=l_{a} H\left(t-\tau_{a}\right)$ pour présenter les principes de base de la méthode. Ainsi dans le domaine fonctionnel (en gardant les mêmes notations pour les signaux et les distributions associées), le système en défaut satisfait l'équation suivante :

$$
a_{1} y^{(1)}+a_{0} y=a_{1} y(0) \delta+b u+b l_{a} H\left(t-\tau_{a}\right)
$$


L'algorithme de diagnostic proposé se compose de deux étapes. La première étape consiste à générer des relations de redondance et la seconde à analyser ces relations. Pour la génération des relations de redondance, l'équation du système en défaut [8] est dérivée dans un premier temps, ce qui donne :

$$
a_{1} y^{(2)}+a_{0} y^{(1)}-b u^{(1)}=\phi_{0}+b l_{a} \delta_{\tau_{a}}
$$

avec

$$
\phi_{0}=a_{1} y(0) \dot{\delta}+\left(a_{1} \dot{y}(0)+a_{0} y(0)-b u(0)\right) \delta
$$

On a

1) $\operatorname{Supp}\left(\phi_{0}\right)=\{0\}$ et $\operatorname{Ordre}\left(\phi_{0}\right)=1$. Ainsi $t^{k_{1}} \phi_{0}=0, \forall k_{1} \geq 2$

2) $\operatorname{Supp}\left(\delta_{\tau_{a}}\right)=\left\{\tau_{a}\right\}$ et $\operatorname{Ordre}\left(\delta_{\tau_{a}}\right)=0$. Ainsi $\left(t-\tau_{a}\right)^{k_{2}} \delta_{\tau_{a}}=0, \forall k_{2} \geq 1$

Ainsi, pour éliminer les singularités du second membre de l'équation ci-dessus ( $\phi_{0}$ et $\delta_{\tau_{a}}$ ), il suffit, d'après le théorème (1), de multiplier l'égalité [9] par une fonction régulière $\alpha_{1}$ vérifiant

$$
\alpha_{1}(0)=\dot{\alpha}_{1}(0)=\alpha_{1}\left(\tau_{a}\right)=0
$$

Soit par exemple, en choisissant ci-dessus $k_{1}=2$ et $k_{2}=1$

$$
\alpha_{1}(t)=t^{2}\left(t-\tau_{a}\right)
$$

On obtient l'égalité

$$
\left[t^{3}\left(a_{1} y^{(2)}+a_{0} y^{(1)}-b u^{(1)}\right)\right]-\tau_{a}\left[t^{2}\left(a_{1} y^{(2)}+a_{0} y^{(1)}-b u^{(1)}\right)\right]=0
$$

Ou encore

$$
\left[\sum_{j=0}^{1} a_{j} W_{2, j}-b V_{2}\right]-\tau_{a}\left[\sum_{j=0}^{1} a_{j} W_{1, j}-b V_{1}\right]=0
$$

avec

$$
\text { pour } i=1,2 \text { et } j=0,1, \begin{cases}W_{i, j} & =t^{1+i} y^{(j+1)} \\ V_{i} & =t^{1+i} u^{(1)}\end{cases}
$$

Pour $t<\tau_{a}$, l'égalité [13] est vérifiée indépendamment de $\tau_{a}\left(\tau_{a}\right.$ n'est pas identifiable avant l'apparition du défaut). En effet pour $t<\tau_{a}$, on montre que

$$
\left[\sum_{j=0}^{1} a_{j} W_{2, j}-b V_{2}\right]=\left[\sum_{j=0}^{1} a_{j} W_{1, j}-b V_{1}\right]=0
$$


Cependant pour $t \geq \tau_{a}$, l'application d'intégrations successives permet d'obtenir des relations de redondance que l'on peut arranger afin d'obtenir la formulation spectrale suivante

$$
\left[A_{2}-\tau_{a} A_{1}\right] X=0
$$

où les matrices $A_{i}$ de dimension $m \times 3(m$ paramètre à choisir en fonction de contraintes explicitées ultérieurement) sont parfaitement connues en fonction des mesures $u$ et $y$. Quant au vecteur $X$, il regroupe tous les paramètres que l'on ne souhaite pas estimer $\left(a_{1}, a_{0}\right.$ et $\left.b\right)$. Plus précisément la $j^{e}$ ligne de la matrice $A_{i}(j=1, \cdots, m)$ est donnée par

$$
(\underbrace{\int_{0}^{t} \cdots \int}_{p+j \text { fois }} \tau^{i+1} \ddot{y}(\tau) d \tau^{p+j} \underbrace{\int_{0}^{t} \cdots \int}_{p+j \text { fois }} \tau^{i+1} \dot{y}(\tau) d \tau^{p+j}-\underbrace{\int_{0}^{t} \cdots \int}_{p+j \text { fois }} \tau^{i+1} \dot{u}(\tau) d \tau^{p+j})
$$

ou encore en fonction de produits de convolution

$$
\begin{gathered}
A_{i}(j,:)=\left(\begin{array}{lll}
H^{\star p+j} \star W_{i, 1} & H^{\star p+j} \star W_{i, 0} & -H^{\star p+j} \star V_{i}
\end{array}\right) \\
\text { et } \quad X=\left(\begin{array}{lll}
a_{1} & a_{0} & b
\end{array}\right)^{T}
\end{gathered}
$$

Afin d'éliminer dans $A_{i}$ toutes les dérivations et de ne faire apparaître que des intégrations qui sont plus robustes numériquement, il suffit de prendre $p \geq 1$. En effet, d'après le théorème (1) et l'égalité [1], $W_{i, j}$ et $V_{i}$ peuvent être exprimés en fonction des dérivées (d'ordre maximal 2) des termes $t^{k} y$ et $t^{k} u$ respectivement, par exemple

$$
W_{2,1}=t^{3} y^{(2)}=t^{3}\left[\delta^{(2)} \star y\right]=\sum_{i=0}^{2}(-1)^{i} \frac{2 !}{(2-i) !}\left(t^{3-i} y\right)^{(2-i)}
$$

À partir de l'égalité [16], $\tau_{a}$ peut être considéré comme une valeur propre généralisée sans qu'il ne soit nécessaire d'estimer le vecteur $X$ ((Wright et al., 2002), (Boutry et al., 2005)).

Pour estimer l'amplitude $l_{a}$, on multiple dans un premier temps l'équation [9] par une fonction régulière $\alpha_{2}$ vérifiant $\alpha_{2}(0)=\dot{\alpha}_{2}(0)=0$ et $\alpha_{2}\left(\tau_{a}\right) \neq 0$ afin d'avoir

$$
a_{1} \alpha_{2} y^{(2)}+a_{0} \alpha_{2} y^{(1)}-b \alpha_{2} u^{(1)}=b l_{a} \alpha_{2}\left(\tau_{a}\right) \delta_{\tau_{a}}
$$

À partir de l'égalité ci-dessus, l'estimation de l'amplitude $l_{a}$ peut être obtenue en fonction de celle de $\tau_{a}$. Cette estimation est donnée par exemple en considérant la fonction $\alpha_{2}=t^{2}$, par

$$
l_{a}=p ! \frac{A_{i}(1,:) \theta}{\tau_{a}^{i+1}\left(t-\tau_{a}\right)^{p}}
$$

$\theta$ est le vecteur propre généralisé de $\left(A_{2}, A_{1}\right)$ associé à $\tau_{a}$, avec $\theta_{1}=1$. 
Dans l'égalité [20] ainsi que dans la suite de cet article, à condition que cela ne prête pas à confusion, on utilise les mêmes notations pour les valeurs exactes $\left(l_{a}, \tau_{a} \ldots\right)$ et leurs estimées. La logique de décision se base sur l'étude de la stationnarité des estimées. Ainsi, on peut conclure de l'apparition d'un défaut actionneur $f_{a}=l_{a} H\left(t-\tau_{a}\right)$ lorsque les estimées de $\tau_{a}$ et $l_{a}$ deviennent simultanément stationnaires à partir de $t \geq \tau_{a}$. Les figures 3 à 5 illustrent des résultats de simulation dans le cas sans bruit.

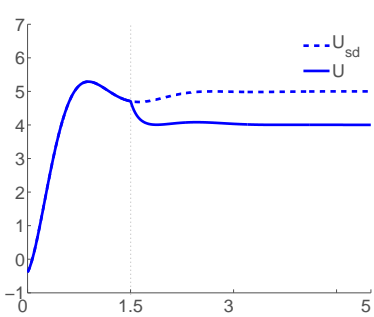

Figure 3. Commande

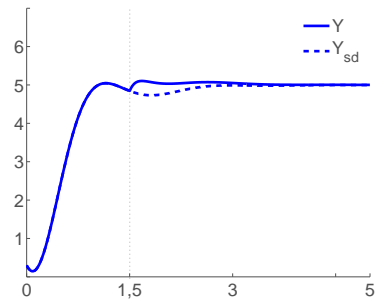

Figure 4. Sortie

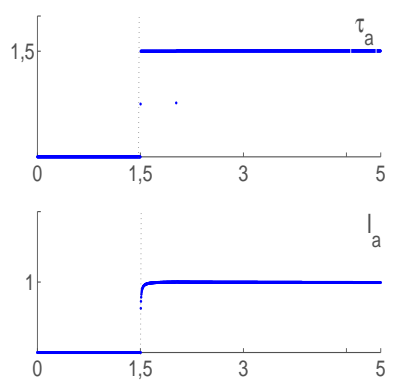

Figure 5. $\tau_{a}$ (en haut) et $l_{a}$ (en bas)

Le système est commandé en boucle fermée à l'aide d'un correcteur PI. L'entrée et la sortie obtenues sans défaut $\left(U_{s d}, U_{s d}\right)$ et avec défaut $(U, Y)$ sont présentées sur les figures 3 et 4 Le défaut actionneur d'amplitude constante $l_{a}=1$ apparaît à l'instant $\tau_{a}=1,5$. Le défaut simulé est bien détecté à travers l'évolution temporelle des estimées de $\tau_{a}$ et $l_{a}$ (figure 5). Lorsque les quantités estimées sont des valeurs aberrantes, c'est-à-dire déviant nettement du comportement de stationnarité (fluctuations par exemple), nous considérons que le défaut n'est pas apparu et fixons les valeurs estimées à zéro. 


\subsection{Système avec défaut capteur}

Nous reprenons l'étude faite dans la section précédente en considérant cette fois le système en présence non pas d'un défaut actionneur, mais d'une dérive capteur apparue à l'instant $\tau_{c}$ avec une pente $l_{c}$. Un tel système est représenté par

$$
a_{1} y^{(1)}+a_{0} y=a_{1} y(0) \delta+b u+l_{c}\left(a_{1} H\left(t-\tau_{c}\right)+a_{0}\left(t-\tau_{c}\right) H\left(t-\tau_{c}\right)\right)
$$

L'application de deux dérivations donne

$$
a_{1} y^{(3)}+a_{0} y^{(2)}-b u^{(2)}=\phi_{0}+l_{c}\left(a_{1} \delta_{\tau_{c}}^{(1)}+a_{0} \delta_{\tau_{c}}\right)
$$

Pour éliminer les singularités apparues au second membre de l'équation ci-dessus, il suffit de multiplier cette dernière par la fonction polynomiale (donc régulière)

$$
\alpha_{3}(t)=t^{3}\left(t-\tau_{c}\right)^{2}=t^{5}-2 \tau_{c} t^{4}+\tau_{c}^{2} t^{3}
$$

Cette fonction vérifie bien $\alpha_{3}(0)=\dot{\alpha}_{3}(0)=\ddot{\alpha}_{3}(0)=\alpha_{3}\left(\tau_{c}\right)=\dot{\alpha}_{3}\left(\tau_{c}\right)=0$ et comme

1) $\operatorname{Supp}\left(\phi_{0}\right)=\{0\}$ et $\operatorname{Ordre}\left(\phi_{0}\right)=2$, alors $t^{k_{1}} \phi_{0}=0, \forall k_{1} \geq 3$

2) $\operatorname{Supp}\left(\delta_{\tau_{c}}^{(1)}\right)=\left\{\tau_{c}\right\}$ et $\operatorname{Ordre}\left(\delta_{\tau_{c}}^{(1)}\right)=1$, alors $\left(t-\tau_{c}\right)^{k_{3}} \delta_{\tau_{c}}=0, \forall k_{3} \geq 2$

Ainsi, d'après le théorème (1), on a

$$
\alpha_{3}\left[a_{1} y^{(3)}+a_{0} y^{(2)}-b u^{(2)}\right]=0
$$

ou encore, après développement

$$
\left[\sum_{j=0}^{1} a_{j} W_{3, j}-b V_{3}\right]-2 \tau_{c}\left[\sum_{j=0}^{1} a_{j} W_{2, j}-b V_{2}\right]+\tau_{c}^{2}\left[\sum_{j=0}^{1} a_{j} W_{1, j}-b V_{1}\right]=0
$$

avec $W_{i, j}=t^{2+i} y^{(2+j)}$ et $V_{i}=t^{2+i} u^{(2)}$ pour $i=1,2,3$ et $j=0,1$.

Pour $t<\tau_{c}$, comme précédemment, on montre que l'égalité ci-dessus est indépendante de $\tau_{c}$ et pour $t \geq \tau_{c}$, l'application d'intégrations successives permet d'obtenir l'égalité algébrique suivante :

$$
\left[A_{4}-2 \tau_{c} A_{3}+\tau_{c}^{2} A_{2}\right] X=0
$$

avec $A_{i}$, pour $i=2,3,4$ défini par [17] et $X$ contenant les paramètres du système. Afin d'estimer $\tau_{c}$ comme valeur propre généralisée d'un couple de matrices, une formulation de type [16] est recherchée. Elle est obtenue en multipliant l'équation [26] par une matrice $B$ non nulle vérifiant $B A_{4} \neq 0$ et

$$
\left\{\begin{array} { l } 
{ B A _ { 2 } = 0 } \\
{ B A _ { 3 } \neq 0 }
\end{array} \text { ou } \left\{\begin{array}{l}
B A_{3}=0 \\
B A_{2} \neq 0
\end{array}\right.\right.
$$


puisque l'on a, selon le cas

$$
\left[B A_{4}-2 \tau_{c} B A_{3}\right] X=0 \text { ou }\left[B A_{4}+\tau_{c}^{2} B A_{2}\right] X=0
$$

Rappelons qu'une condition suffisante de l'existence de la matrice $B$ est que les matrices $A_{i}$ soient rectangulaires verticales $(m>3)$.

À partir des égalités en [28], $\tau_{c}$ peut être estimée soit directement (comme valeur propre généralisée de $\left(B A_{4}, 2 B A_{3}\right)$ ) soit en estimant d'abord $\tau_{c}^{2}$ (valeur propre généralisée de $\left.\left(B A_{4}, B A_{2}\right)\right)$. Par la suite, l'amplitude $l_{c}$ peut être estimée en multipliant d'abord l'équation [22] par une fonction régulière $\alpha_{4}$ vérifiant

$$
\left\{\begin{array}{l}
\alpha_{4}(0)=\dot{\alpha}_{4}(0)=\ddot{\alpha}_{4}(0)=\alpha_{4}\left(\tau_{c}\right)=0 \\
\dot{\alpha}_{4}\left(\tau_{c}\right) \neq 0
\end{array}\right.
$$

ce qui conduit à l'égalité

$$
\alpha_{4}\left[a_{1} y^{(3)}+a_{0} y^{(2)}-b u^{(2)}\right]=l_{c} a_{1} \alpha_{4} \delta_{\tau_{c}}^{(1)}
$$

or $\alpha_{4} \delta_{\tau_{c}}^{(1)}=-\dot{\alpha}_{4}\left(\tau_{c}\right) \delta_{\tau_{c}}$ d'où

$$
\alpha_{4}\left[a_{1} y^{(3)}+a_{0} y^{(2)}-b u^{(2)}\right]=-l_{c} a_{1} \dot{\alpha}_{4}\left(\tau_{c}\right) \delta_{\tau_{c}}
$$

Ainsi, en considérant la fonction $\alpha_{4}=t^{3}\left(t-\tau_{c}\right)$, on obtient l'estimation de l'amplitude $l_{c}$ par

$$
l_{c}=p ! \frac{\left(A_{3}(1,:)-\tau_{c} A_{2}(1,:)\right) \theta}{\theta_{1} \tau_{c}^{3}\left(t-\tau_{c}\right)^{p}}
$$

$\theta$ est le vecteur propre généralisé de $\left(A_{2}, A_{1}\right)$ associé à $\tau_{a}$, avec $\theta_{1}=1$.

La logique de décision est la même que pour le défaut actionneur. La détection du défaut est accomplie lorsque les estimations de $\tau_{c}$ et $l_{c}$ deviennent stationnaires simultanément après l'apparition du défaut. L'efficacité de l'approche de diagnostic présentée en présence d'une dérive capteur est illustrée sur les figures 6 à 8

Le système est commandé en boucle fermée à l'aide d'un correcteur PI. L'entrée et la sortie obtenues sans défaut $\left(u_{s d}, y_{s d}\right)$ et avec défaut $(u, y)$ sont présentées sur les figures 6 et 7 Le défaut capteur de type rampe de pente $l_{c}=-0,3$ apparaît à l'instant $\tau_{c}=1,5$. Le défaut simulé est bien détecté à travers l'évolution temporelle des estimées de $\tau_{c}$ et $l_{c}$ (figure 8). On peut aussi noter une plus grande sensibilité offerte par la technique utilisée pour la détection du défaut de type rampe avec une faible pente. 


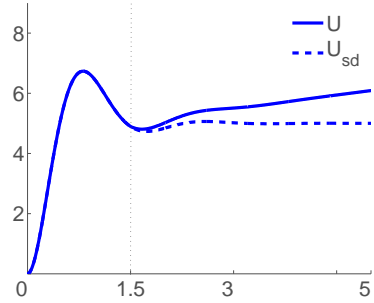

Figure 6. Commande

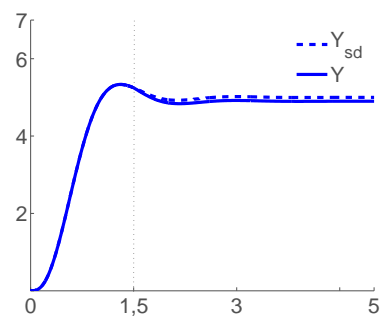

Figure 7. Sortie

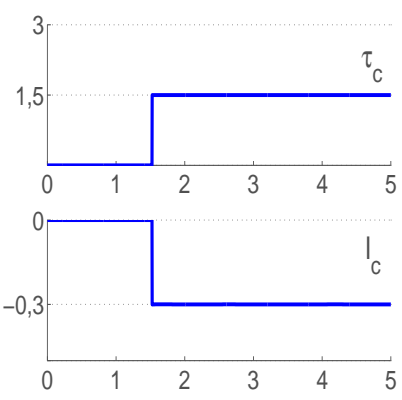

Figure 8. $\tau_{c}$ (en haut) et $l_{c}$ (en bas)

\subsection{Système avec défaut actionneur et défaut capteur}

Dans le cas de défauts multiples (actionneur et capteur), la seule application des démarches précédentes n'assure pas la détection et la localisation des défauts. Considérons le cas simple d'un système du premier ordre en présence d'un biais actionneur et d'un biais capteur modélisés respectivement par

$$
f_{a}=l_{a} H\left(t-\tau_{a}\right) \text { et } f_{c}=l_{c} H\left(t-\tau_{c}\right)
$$

Le modèle du système en défaut est donné par

$$
a_{1} y^{(1)}+a_{0} y=\phi_{0}+b u+b l_{a} H\left(t-\tau_{a}\right)+l_{c}\left(a_{1} \delta_{\tau_{c}}+a_{0} H\left(t-\tau_{c}\right)\right)
$$

L'application d'une dérivation sur ce dernier donne

$$
a_{1} y^{(2)}+a_{0} y^{(1)}-b u^{(1)}=\phi_{0}^{(1)}+b l_{a} \delta_{\tau_{a}}+l_{c}\left(a_{1} \delta_{\tau_{c}}^{(1)}+a_{0} \delta_{\tau_{c}}\right)
$$

et la multiplication par $\alpha_{1}=t^{2}\left(t-\tau_{a}\right)$ ou $\alpha_{3}=t^{2}\left(t-\tau_{c}\right)^{2}$ de l'équation 34 n'annule pas toutes les singularités apparues. Pour y parvenir, il suffit de considérer une fonction régulière $\alpha_{5}$ satisfaisant

$$
\alpha_{5}(0)=\alpha_{5}\left(\tau_{a}\right)=\alpha_{5}\left(\tau_{c}\right)=0 \quad \text { et } \quad \alpha_{5}^{(1)}(0)=\alpha_{5}^{(1)}\left(\tau_{c}\right)=0
$$


Le choix de $\alpha_{5}(t)=t^{2}\left(t-\tau_{a}\right)\left(t-\tau_{c}\right)^{2}$ permet d'obtenir la formulation spectrale

$$
\left[A_{4}-\left(\tau_{a}+2 \tau_{c}\right) A_{3}+\left(2 \tau_{a} \tau_{c}+\tau_{c}^{2}\right) A_{2}-\left(\tau_{a} \tau_{c}^{2}\right) A_{1}\right] X=0
$$

Pour la détection et la localisation des différents défauts dans ce cas, le système de diagnostic que nous proposons est composé de trois cellules. La première consiste à la détection du défaut actionneur unique (section 3.2), la seconde à la détection du défaut capteur unique (section 3.3) et la troisième qui s'obtient avec $\alpha_{5}$ et qui consiste à détecter les défauts multiples.

Ainsi le premier défaut peut être détecté à l'aide de la cellule qui lui est associée et ceci avant l'apparition du second défaut. Ce dernier ne peut pas être détecté au moyen de la cellule qui lui est associée à cause du premier défaut apparu. En revanche, la troisième cellule permet cette détection. En effet, considérons la matrice $C$ non nulle vérifiant

$$
C\left[\begin{array}{ll}
A_{2} & A_{1}
\end{array}\right]=0
$$

à partir de l'équation [36, on obtient l'égalité

$$
\left[C A_{4}-\left(\tau_{a}+2 \tau_{c}\right) C A_{3}\right] X=0
$$

qui permet d'obtenir une estimation de la combinaison $\tau_{a}+2 \tau_{c}$ à l'aide des valeurs propres généralisées de $\left(C A_{4}, C A_{3}\right)$. Cette estimation devient stationnaire à partir de l'instant $t \geq \max \left(\tau_{a}, \tau_{c}\right)$. Notons enfin que l'amplitude du second défaut peut être estimée en fonction des estimations de $\tau_{a}$ et $\tau_{c}$. La logique de décision est la même que dans le cas d'un défaut unique, c'est-à-dire le second défaut est détecté au moyen de la stationnarité simultanée des estimées de $\tau_{a}+2 \tau_{c}$ et $l_{c}$.

Les résultats de simulation sont représentés graphiquement sur les figures 9 à 12 Le système est commandé à l'aide d'un correcteur $P I$. Les défauts actionneur et capteur d'amplitudes respectives $l_{a}=1$ et $l_{c}=0,5$ apparaissent respectivement aux instants $\tau_{a}=1$ et $\tau_{c}=2$. Comme il l'a été mentionné ci-dessus, le défaut actionneur, apparu en premier, peut être détecté à partir des estimations de $\tau_{a}$ et $l_{a}$ (figure 11). À partir de l'instant $t>2$, l'estimation de $\tau_{a}$ fluctue du fait de l'apparition du défaut capteur à cet instant. Notons que le défaut capteur ne peut pas être détecté à partir des estimations de $\tau_{c}$ et $l_{c}$ (car lors de son apparition, le système présente déjà un défaut actionneur). En revanche ce dernier peut être détecté à partir des estimations de $\tau_{a}+2 \tau_{c}$ et $l_{c}$ (obtenue en fonction des estimations de $\tau_{a}+2 \tau_{c}$ ) représentées sur la figure 12 


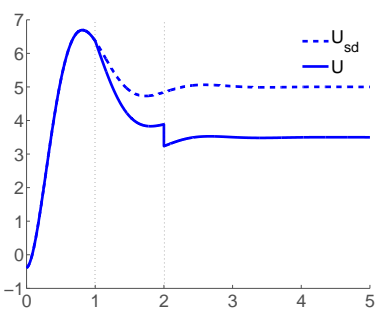

Figure 9. Commande

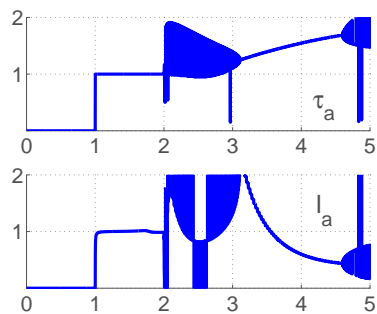

Figure 11. Estimations de $\tau_{a}$ et $l_{a}$ obtenues avec la cellule de détection de défaut actionneur

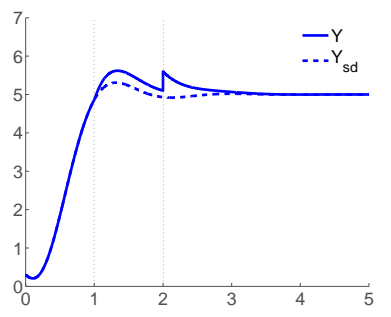

Figure 10. Sortie

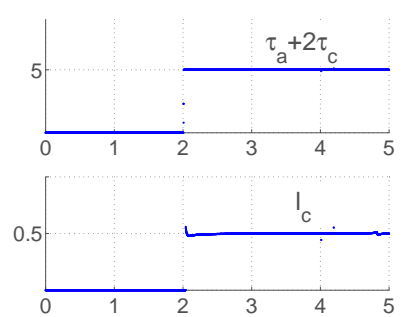

Figure 12. Estimations de $\tau_{a}+2 \tau_{c}$ et $l_{c}$ obtenues avec la cellule de détection de défauts multiples

Remarque 1 (Défauts non localisables)

L'algorithme de diagnostic présenté permet toujours la détection de défaut. Cependant, pour des structures de défaut particulières, la localisation n'est pas toujours possible. En effet, si l'on considère l'exemple du premier ordre précédent, les défauts actionneurs

$$
f_{a}(t)=l_{a}\left[H\left(t-\tau_{a_{1}}\right)-H\left(t-\tau_{a_{2}}\right)\right]
$$

et capteurs $f_{c}(t)$ solution de l'équation

$$
a_{1} f_{c}^{(1)}+a_{0} f_{c}=-b l_{c} H\left(t-\tau_{c}\right)
$$

auront les même signatures sur u et y qu'un défaut actionneur unique

$$
f_{a}(t)=l_{a}\left[H\left(t-\tau_{a_{1}}\right)-H\left(t-\tau_{a_{2}}\right)\right]-l_{c} H\left(t-\tau_{c}\right)
$$

Dans ce cas, il est impossible de distinguer la situation de défauts multiples de celle d'un défaut unique.

Précisons que cette remarque n'est pas spécifique à la méthode proposée. 


\subsection{Cas général}

On se situe dans le formalisme des distributions avec des descriptions entrées/sorties en supposant que la commande $u_{r}$ et la sortie $y_{r}$ du système étudié vérifient une équation de la forme

$$
a_{n} y_{r}^{(n)}+a_{n-1} y_{r}^{(n-1)}+\cdots+a_{0} y_{r}=\phi_{0}+b u_{r}
$$

où $n$ est supposé connu et les paramètres $a_{i}$ et $b$ sont supposés constants mais pas nécessairement connus. La distribution $\phi_{0}$ est une combinaison linéaire des dérivées d'ordre inférieur ou égal à $n-1$ de la distribution de Dirac $(\delta)$ et contient les conditions initiales (pas nécessairement connues).

En présence d'un défaut actionneur noté $f_{a}$ et/ou d'un défaut capteur noté $f_{c}$, l'équation du système en défaut s'écrit

$$
a_{n} y^{(n)}+\cdots+a_{0} y-b u=\phi_{0} b f_{a}+a_{n} f_{c}^{(n)}+\cdots+a_{0} f_{c}
$$

La tâche de diagnostic de défaut est basée sur l'idée que des termes inconnus (caractérisant les défauts) peuvent être estimés à partir de la seule connaissance de la structure du système et des signaux. Lorsque les signaux défauts $f_{a}$ et/ou $f_{c}$ sont structurés et après avoir choisi et appliqué sur le modèle du système en défaut les annulateurs des termes structurés (conditions initiales, défauts)

$$
\Gamma=\Gamma_{k}-\beta_{k-1} \Gamma_{k-1}-\cdots-\beta_{1} \Gamma_{1}
$$

on obtient une égalité fonctionnelle à partir de laquelle l'application d'intégrations successives permet de se ramener à un problème du type

$$
\left[A_{k}-\beta_{k-1} A_{k-1}-\cdots-\beta_{1} A_{1}\right] X=0
$$

où

- les matrices $A_{i}$ de dimension $m \times(n+2)$ sont totalement définies à partir des mesures $u$ et $y$ et données par :

$$
\begin{aligned}
A_{i}(j, \mu) & =\underbrace{\int_{0}^{t} \cdots \int}_{p+j \text { times }} \Gamma_{i} y^{(n-\mu+1)} d \tau^{p+j}, \mu=1, \cdots, n+1 \\
A_{i}(j, n+2) & =-\underbrace{\int_{0}^{t} \cdots \int}_{p+j \text { times }} \Gamma_{i} u d \tau^{p+j}
\end{aligned}
$$

Le polynôme différentiel [44] est écrit sous forme d'une combinaison linéaire de monômes différentiels élémentaires donnés par

$$
\Gamma_{j}=t^{n_{j}} \frac{d^{d_{j}}}{d t^{d_{j}}}, n_{j}, d_{j} \in \mathbb{N}
$$


Le nombre des redondances $m$ est choisi en fonction de certaines contraintes liées à la structure du système et celle des défauts. En choisissant le paramètre $p \geq n+d$, où $d$ est l'ordre de dérivation le plus élevé apparaissant dans $\Gamma$, les intégrations successives initiales assurent l'élimination de toutes les dérivées dans l'écriture des matrices $A_{i}$.

- les paramètres $\beta_{j}$ contiennent les informations sur les défauts.

- $X$ contient les paramètres du système dont l'estimation n'est pas nécessaire, soit encore

$$
X=\left(\begin{array}{lllll}
a_{n} & a_{n-1} & \cdots & a_{0} & b
\end{array}\right)^{T} \in \mathbb{R}^{n+2}
$$

Estimer $\beta_{j}(j=1, \cdots, k-1)$ revient alors à déterminer une des valeurs propres généralisées du couple de produits de matrices $\left(B A_{k}, B A_{j}\right)$ où $B$ est une matrice non nulle vérifiant :

$$
B A_{i}=0, \forall i \neq j
$$

Les matrices $A_{i}$ ne sont pas stationnaires mais varient avec le temps, par conséquent les valeurs propres généralisées de $\left(B A_{k}, B A_{j}\right)$ évoluent aussi avec le temps. Parmi toutes les valeurs propres généralisées possibles de $\left(B A_{k}, B A_{j}\right)$, on choisit celle ayant un comportement stationnaire sur un horizon de temps.

\section{Remarque 2 Existence de la matrice $B$.}

1) La matrice B peut facilement être obtenue à l'aide de la décomposition $Q R$.

2) Les paramètres $\beta_{j}$ étant au nombre de $k-1$ et les matrices $A_{i}$ de dimension $m \times(n+2)$, le paramètre $m$ doit être choisi tel que $m>(k-2)(n+2)$.

Après l'estimation d'un certain nombre de composantes du vecteur $\beta$, on peut, en fonction de ces dernières, estimer les autres paramètres associés aux défauts (amplitude, pente...).

À l'instar de tout algorithme de diagnostic, la prise de décision est fondée sur l'évolution temporelle des estimations. Un défaut est détecté et localisé lorsque les estimations de ses paramètres deviennent simultanément stationnaires.

La méthode algébrique de diagnostic de défauts présentée dans cette section ne requiert que la seule connaissance de la structure du système et des signaux pour être mise en œuvre. Le développement de la démarche par la technique d'identification algébro-différentielle est simple puisque l'utilisation uniquement des mesures de la commande et de la sortie du système ainsi que la connaissance de l'ordre de ce dernier suffit à obtenir des résultats utiles au diagnostic.

L'extension de l'approche au système multivariable est évidente en partant du fait que la représentation «entrées - sorties » d'un tel système peut être décomposée en plusieurs systèmes à plusieurs entrées et une sortie. 


\section{Etude de la robustesse de l'approche}

\subsection{Robustesse par rapport à l'ordre du sytème}

Le paramètre le plus important à connaître a priori pour appliquer l'algorithme développé est l'ordre du système. Il est donc primordial d'examiner le comportement des algorithmes de diagnostic du système lors d'une sur-modélisation ou sousmodélisation du système. Notons qu'il existe de nombreuses méthodes dans le domaine de l'identification des systèmes pour estimer l'ordre d'un système (Ljung, 1999).

Pour examiner l'influence de l'ordre du système sur l'approche proposée, considérons un système d'ordre exact $N$ et $n$ le prérequis. En présence d'un défaut actionneur noté $f_{a}$ et d'un défaut capteur noté $f_{c}$, on obtient pour le système $\left(\sum\right)$ et le modèle $(M)$ les représentations suivantes :

$$
\begin{gathered}
\left(\sum\right): \tilde{a}_{N} y^{(N)}+\cdots+\tilde{a}_{0} y=\tilde{b} u+\psi_{0}+\tilde{b} f_{a}+\tilde{a}_{N} f_{c}^{(N)}+\cdots+\tilde{a}_{0} f_{c} \\
(M): a_{n} y^{(n)}+\cdots+a_{0} y=b u+\phi_{0}+b f_{a}+a_{n} f_{c}^{(n)}+\cdots+a_{0} f_{c}+\mathfrak{E}
\end{gathered}
$$

Les condition initiales $\psi_{0}$ et $\phi_{0}$ de support commun $\{0\}$ et d'ordre respectif $N-1$ et $n-1$ peuvent être écrites comme :

$$
\begin{aligned}
& \psi_{0}(t)=\sum_{k=1}^{N} \tilde{a}_{k} \sum_{i=0}^{k-1} y^{(i)}(0) \delta^{(k-i-1)} \\
& \phi_{0}(t)=\sum_{k=1}^{n} a_{k} \sum_{i=0}^{k-1} y^{(i)}(0) \delta^{(k-i-1)}
\end{aligned}
$$

E contient l'erreur de modélisation telle que les équations [51] et [52] soient cohérentes.

Dans une situation de modélisation exacte, c'est-à-dire lorsque l'ordre $n$ du modèle est équivalent à celui du système étudié $N$, l'algorithme developpé ci-dessus permet d'accomplir la tâche de diagnostic du système en présence de défauts actionneurs et/ou capteurs. La même performance peut être attendue lorsque l'ordre du modèle $n$ est supérieur à celui du système $N$ (sur-estimation). En effet, dans une telle situation, on vérifie facilement que

- l'erreur de modélisation $\mathfrak{E}$ est nulle,

- L'opérateur annulateur de $\phi_{0}, f_{a}$ et $f_{c}^{(n)}$ (dans [52]) annule également les termes $\psi_{0}, f_{a}$ et $f_{c}^{(N)}$ (dans [51]). 
Ainsi, à travers les différentes étapes de l'approche proposée, on obtient un problème du type [45], autrement dit, on obtient des informations utiles au diagnostic.

Cependant, lorsque l'ordre du système étudié est sous-estimé, c'est-à-dire $N>n$, alors une égalité du type [45] n'existe plus. On aura en revanche :

$$
\left[A_{k}-\beta_{k-1} A_{k-1}-\cdots-\beta_{1} A_{1}\right] X=R \neq 0
$$

où l'élément $j(j=1, \cdots, m)$ du vecteur des résidus $R$ est donné par :

$$
R(j)=\sum_{k=n+1}^{N} \tilde{a}_{k} \underbrace{\int_{0}^{t} \cdots \int}_{p+j \text { times }} \Gamma\left(\left(-y+f_{c}\right)^{(k)}+\sum_{i=0}^{k-(n+1)} y^{(i)}(0) \delta^{(k-1-i)}\right)
$$

En effet, l'annulateur $\Gamma$ des termes $\phi_{0}, f_{a}$ et $f_{c}^{(n)}$ apparaissant dans [52] vérifie

$$
\begin{aligned}
\Gamma f_{c}^{(i)} & =0, \forall i \leq n \\
\Gamma \psi_{0} & =\sum_{k=n+1}^{N} \sum_{i=0}^{k-(n+1)} y^{(i)}(0) \delta^{(k-1-i)}
\end{aligned}
$$

Le vecteur résidu est une fonction de certaines conditions initiales et des dérivées d'ordre supérieur de $y$ et $f_{c}$ (dans le cas d'un défaut capteur). Lorsque la différence d'ordre est importante alors l'approche proposée peut ne pas identifier le défaut apparu sur le système.

\subsection{Robustesse par rapport au bruit de mesure}

Nous étudions dans cette partie la robustesse de l'approche par rapport aux dynamiques hautes fréquences.

Soit un système dons les grandeurs réelles satisfont l'équation [42]. En plus des défauts $f_{a}$ et $f_{c}$, si on ajoute à la sortie $y$ une perturbation, notée $\pi$, non structurée (c'est le cas par exemple d'un signal à fluctuation rapide), l'équation du système en défaut devient :

$$
a_{n}\left(y+\pi-f_{c}\right)^{(n)}+\cdots+a_{0}(y+\pi-f c)=\phi_{0}+b\left(u+f_{a}\right)
$$

Après la multiplication de l'équation ci-dessus par l'annulateur $\Gamma$ approprié des termes structurés (conditions initiales, défauts) et la génération des relations de redondance par intégrations successives, on obtient :

$$
\left[\left(A_{k}-\Delta A_{k}\right)-\beta_{k-1}\left(A_{k-1}-\Delta A_{k-1}\right)-\cdots-\beta_{1}\left(A_{1}-\Delta A_{1}\right)\right] X=0
$$


Les matrices $A_{i}$ s'expriment en fonction des signaux connus $u$ et $y$ tandis que les matrices $\Delta A_{i}$ sont liées à la perturbation $\pi$.

L'analyse de la robustesse s'appuie tout d'abord sur l'analyse des filtres générateurs des éléments des matrices $A_{i}$. Supposons que l'annulateur des termes structurés (conditions initiales, défauts) puisse s'écrire

$$
\Gamma=t^{n_{k}} \frac{d^{d_{k}}}{d t^{d_{k}}}-\beta_{k-1} t^{n_{k-1}} \frac{d^{d_{k-1}}}{d t^{d_{k-1}}}-\cdots-\beta_{1} t^{n_{1}} \frac{d^{d_{1}}}{d t^{d_{1}}}, n_{j}, d_{j} \in \mathbb{N}
$$

En considérant la formule de Cauchy pour les intégrations multiples 1 , les expressions des matrices $A_{i}$ peuvent être réduites à

$$
\begin{aligned}
A_{i}(j, \mu) & =\int_{0}^{t} f_{i, j, \mu}(t-\tau) y(\tau) d \tau, \mu=1, \cdots, n+1 \\
A_{i}(j, n+2) & =\int_{0}^{t} g_{i, j}(t-\tau) u(\tau) d \tau
\end{aligned}
$$

où $f_{i, j, \mu}$ et $g_{i, j}$ sont des polynômes de degrés $p+j+\mu+n_{i}-n-d_{i}-2$ et $p+j+n_{i}-d_{i}-1$ respectivement. Pour l'exemple de la sous-section 3.2 avec $\Gamma=t^{2}\left(t-\tau_{a}\right) \frac{d}{d t}$, les polynômes correspondants sont caractérisés par :

$$
\begin{aligned}
& f_{i, j, 1}(\tau)=\frac{\tau^{p+j-3}(t-\tau)^{i+1}}{(p+j-3) !}-\frac{2(i+1) \tau^{p+j-2}(t-\tau)^{i}}{(p+j-2) !}+\frac{i(i+1) \tau^{p+j-1}(t-\tau)^{i-1}}{(p+j-1) !} \\
& f_{i, j, 2}(\tau)=\frac{\tau^{p+j-2}(t-\tau)^{i+1}}{(p+j-2) !}-\frac{(i+1) \tau^{p+j-1}(t-\tau)^{i}}{(p+j-1) !} \\
& g_{i, j}(\tau)=f_{i, j, 2}(\tau)
\end{aligned}
$$

Ainsi, la matrice de transfert entre $e=\left[\begin{array}{ll}u & y\end{array}\right]^{T}$ et $A_{i}(j,:)$ considérée comme un filtre causal passe-bas, c'est-à-dire qui ne «laisse passer » que les basses fréquences, les hautes fréquences (bruits) étant fortement atténuées admet pour réponse impulsionnelle

$$
h(t)=\left[\begin{array}{cccc}
0 & \cdots & 0 & g_{i, j}(t) \\
f_{i, j, 1}(t) & \cdots & f_{i, j, n+1}(t) & 0
\end{array}\right]^{T}
$$

On peut trouver une analyse des performances de ce filtre dans le domaine discret dans (García Collado et al., 2009), où une approximation de l'intégrale basée sur une discrétisation trapézoïdale régulièrement espacée a été utilisée.

1. $\underbrace{\int_{0}^{t} \cdots \int}_{p \text { fois }} f(\tau) d \tau^{p}=\int_{0}^{t} \frac{(t-\tau)^{p-1}}{(p-1) !} f(\tau) d \tau$ 
Un autre outil sur lequel l'analyse de la robustesse peut s'appuyer est le $\epsilon$ pseudospectre d'un faisceau de matrices. Les méthodes traditionnelles de résolution de l'equation [58] ne donnent pas souvent de solution. Les matrices $A_{i}$ données par la procédure de diagnostic correspondent aux matrices $\tilde{A}_{i}$ auxquelles s'ajoutent des perturbations $\Delta A_{i}$ (i.e $A_{i}=\tilde{A}_{i}+\Delta A_{i}$ ). Le mot « faisceau » utilisé en algèbre linéaire fait référence à l'expression $A-\lambda B$, où $A$ et $B$ sont des matrices carrées et $\lambda$ un nombre.

Définition 2 Soit $A$ et $B$ deux matrices de $\mathbb{R}^{m \times n}$. On dit que $\lambda$ est une $\epsilon$-pseudo valeur propre du couple de matrices $(A, B)$, s'il existe un vecteur $\nu$ non nul (le pseudovecteur propre correspondant) tel que

$$
\|(A-\lambda B) \nu\| \leq \epsilon
$$

L'ensemble des $\epsilon$-pseudo valeurs propres de $(A, B)$ est appelé $\epsilon$-pseudospectre de $(A, B)$ et est noté $\Lambda_{\epsilon}(A, B)$.

Notons que si la norme $\|\bullet\|$ considérée est la norme quadratique $\|\bullet\|_{2}$, alors on a la définition équivalente suivante :

$$
\Lambda_{\epsilon}(A, B)=\left\{\lambda \in \mathbb{R}: \sigma_{\min }(A-\lambda B) \leq \epsilon\right\}
$$

Et plus généralement, pour $k$ matrices $A_{i} \in \mathbb{R}^{m \times n}$, on définit l'ensemble

$$
\Lambda_{\epsilon}\left(A_{k}, \cdots, A_{1}\right)=\left\{\beta \in \mathbb{R}^{k-1}: \sigma_{\min }\left(A_{k}-\beta_{k-1} A_{k-1}-. .-\beta_{1} A_{1}\right) \leq \epsilon\right\}
$$

Les ensembles $\Lambda_{\epsilon}\left(A_{k}, \cdots, A_{1}\right)$ sont plus susceptibles d'être vides pour de petites valeurs de $\epsilon$. En revanche, en faisant croître la valeur de $\epsilon$, on obtient une famille d'ensembles $\Lambda_{\epsilon}\left(A_{k}, \cdots, A_{1}\right) \neq\{\emptyset\}$. Ainsi, l'estimation de $\tilde{\beta}$ (solution du problème [58]) peut être obtenue par l'estimation des minima locaux de cette famille d'ensembles. Le cas $k=2$ a déjà fait l'objet de quelques études dans la littérature ((Boutry et al., 2005) et (Wright et al., 2002)) et est connu sous le nom de pseudospectre de faisceaux de matrices. Pour $k>2$, soit $N_{j}$ une matrice orthogonale non nulle de dimension $(m-(k-2) n) \times m$ telle que $N_{j} A_{i}=0$ pour tout $j$ différent de $i$ entre 1 et $k(1 \leq j<k$ et $j \neq i)$. Ainsi, on peut écrire

$$
\begin{array}{r}
\underset{\beta_{j}}{\operatorname{argmin}} \sigma_{\min }\left(A_{k}-\beta_{k-1} A_{k-1}-\cdots-\beta_{1} A_{1}\right)= \\
\underset{\beta_{j}}{\operatorname{argmin}} \sigma_{\min }\left(N_{j} A_{k}-\beta_{j} N_{j} A_{j}\right)
\end{array}
$$

car $\sigma_{\min }$ est invariant par transformation orthogonale. Voir la remarque (2) pour les conditions d'existence des matrices $N_{j}$. 
Un algorithme évident de calcul de $\underset{\lambda}{\operatorname{argmin}} \sigma_{\min }(A-\lambda B)$ pour $A$ et $B$ des matrices de même dimension consiste à évaluer $\sigma_{\min }\left(A-\lambda_{i} B\right)$ pour différents $\lambda_{i}$ et à générer des courbes de niveaux sur lesquelles on relèvera les minima éventuels.

Afin d'illustrer la robustesse de l'approche par rapport aux bruits de mesure, considérons l'exemple de la sous section 3.2 avec la sortie entachée par un bruit additif, gaussien et centré, de variance 0.01 qu'on note $\pi$.

La figure 13 représente la sortie $y$ avec le bruit de mesure $\pi$ simulé.

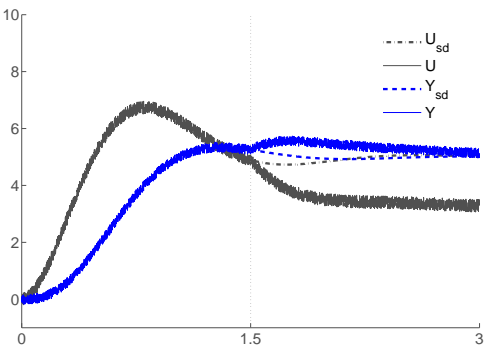

Figure 13. Commande et sortie

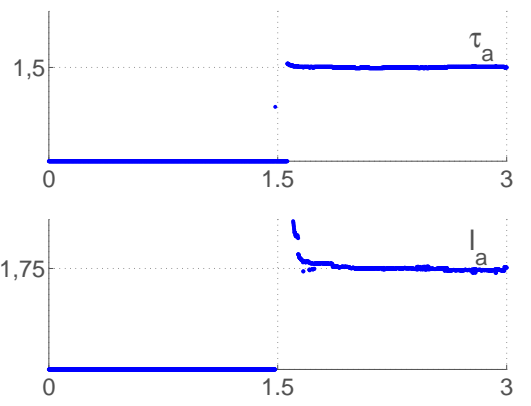

Figure 14. $\tau_{a}$ (en haut) et $l_{a}$ (en bas)

À travers les résultats graphiques représentés sur la figure 14, on peut conclure de l'apparition du défaut actionneur simulé $f_{a}=-H(t-1,5)$ avec cette fois-ci un léger retard de détection. L'analyse de ces courbes confirme la robustesse de l'approche proposée par rapport aux bruits additifs à fluctuations rapides. On trouvera dans (Fliess, 2006) d'autres raisons théoriques expliquant la robustesse à ces bruits se fondant sur l'analyse non standard.

\section{Conclusion}

Dans cet article, le problème de diagnostic des systèmes dynamiques linéaires a été traité , dans le cadre d'une nouvelle théorie déterministe de l'estimation, reposant sur le calcul fonctionnel. Cette théorie est à la base d'une nouvelle méthode de diagnostic que nous avons proposée et qui consiste à estimer directement les paramètres des défauts par simple analyse spectrale. Nous avons focalisé notre étude sur le diagnostic de défauts d'actionneurs et de capteurs pour une classe de systèmes dynamiques continus linéaires incertains. Des algorithmes de détection, localisation et identification de défauts basés sur des propriétés structurelles du système et des défauts ont été développés, en supposant que seuls l'ordre du système et les signaux entrées et sorties sont connus. Les méthodes développées sont appliquées à des exemples académiques afin d'illustrer leur efficacité. Grâce à la non-prise en compte des conditions initiales et à la rapidité des calculs (due aux expressions explicites), le diagnostic sous un angle 
local est envisageable. Ceci permettrait également à l'approche de pouvoir s'appliquer à des systèmes évoluant lentement avec le temps.

Une analyse rigoureuse de la robustesse de la méthode proposée reste à mener plus particulièrement par rapport à l'ordre du système. Il serait intéressant d'inclure dans cette analyse une comparaison avec les méthodes de diagnostic de systèmes dynamiques dites à base de données, notamment l'approche par sous-espace (Ding et al., 2010).

\section{Bibliographie}

Basseville M., Nikiforov I., Detection of abrupt changes : theory and application, Prentice Hall, 1993.

Belkoura L., « Identifiability and algebraic identification of time delay systems », 9th IFAC Workshop on Time Delay System, Prague, Tchèque, 2010.

Boutry G., Elad M., Golub G., Milanfar P., « The generalized eigenvalue problem for nonsquare pencils using a minimal perturbation approach », SIAM Journal on Matrix Analysis and Applications, vol. 27, p. 582-601, 2005.

Ding S. X., Model-Based Fault Diagnosis Techniques - Design Schemes Algorithms and tools, Springer-Verlag, 2008.

Ding S. X., Zhang P., Ding E., Yin E., Naik A., Deng P., Gui W., « On the application of PCA technique to fault diagnosis », Tsinghua Science \& Technology, vol. 15, p. 138-144, 2010.

Fliess M., « Analyse non standard du bruit», Comptes-Rendus de l'Académie des Sciences, Série 1, Mathématiques, vol. 342, p. 797-802, 2006.

Fliess M., Sira-Ramirez H., « An algebraic framework for linear identification », ESAIM Control, Optimization and Calculus of Variations, vol. 9, p. 151-168, 2003.

Frank P., «Fault Diagnosis in Dynamic Systems Using Analytical and Knowledge-based Redundancy - A Survey and Some New Results », Automatica, vol. 26, p. 459-474, 1990.

García Collado F. A., D’Andréa-Novel B., Fliess M., Mounier H., « Analyse fréquentielle des dérivateurs algébriques », XXIIe Colloque GRETSI, Dijon France, 2009.

Gertler J., Cao J., " PCA-based fault diagnosis in the presence of control and dynamics », AIChE Journal, vol. 50, p. 388-402, 2004.

Isermann R., Fault-Diagnosis System, Springer, Berlin, 2006.

Ljung L., System identification (2nd ed.) : theory for the user, Prentice Hall PTR, Upper Saddle River, NJ, USA, 1999.

Patton R., Clark R., Frank P. M., Issues of Fault Diagnosis for Dynamic Systems, SpringerVerlag, London, 2000.

Schwartz L., Théorie des distributions, 2nd edn, Hermann, Paris, 1966.

Staroswiecki M., Gehin A. L., « From control to supervision », Proc. IFAC Safeprocess, p. 312$323,2000$.

Wang J., Qin S. J., « A new subspace identification approach based on principal component analysis », J. of Proc. Control, vol. 12, p. 841-855, 2002. 
Wright T. G., Trefethen L. N., « Pseudospectra of rectangular matrices », IMA J. of Numer. Anal., vol. 22, p. 501-519, 2002. 


\section{ANNEXE POUR LE SERVICE FABRICATION \\ A FOURNIR PAR LES AUTEURS AVEC UN EXEMPLAIRE PAPIER \\ DE LEUR ARTICLE ET LE COPYRIGHT SIGNE PAR COURRIER \\ LE FICHIER PDF CORRESPONDANT SERA ENVOYE PAR E-MAIL}

1. ARTICLE POUR LA REVUE:

JESA - 45/2011. Nouvelles tendances en automatique

2. Auteurs :
A. MOUSSA ALI* - C. JOIN ${ }^{*}, * *-F . H A M E L I N^{*}$

3. TITRE DE L'ARTICLE :

Diagnostic des systèmes dynamiques linéaires sans modèle explicite

4. TITRE ABRÉGÉ POUR LE HAUT DE PAGE MOINS DE 40 SIGNES :

Diagnostic sans modèle explicite

5. DATE DE CETTE Version :

28 novembre 2011

6. COORdonnÉES DES AUTEURS :

- adresse postale :

* CRAN, Nancy-Université, UMR 7039 CNRS, BP 70 239, 54506 Vandœuvre, France

** ALIEN-INRIA Saclay - Ile de France

- téléphone : +33 383684461

- télécopie : +33 383684462

- e-mail : \{amoussaa,cjoin,fhamelin\}@cran.uhp-nancy.fr

7. LOGICIEL UTILISÉ POUR LA PRÉPARATION DE CET ARTICLE :

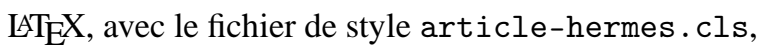
version $1.23 \mathrm{du} 17 / 11 / 2005$.

8. FORMULAIRE DE COPYRIGHT :

Retourner le formulaire de copyright signé par les auteurs, téléchargé sur : http://www.revuesonline.com

SERVICE ÉditoriAL - HERMES-LAVOISIER 14 rue de Provigny, F-94236 Cachan cedex Tél. : 01-47-40-67-67 E-mail : revues@lavoisier.fr Serveur web : http://www.revuesonline.com 